\title{
The Research and Application of heterogeneous database based on XML in Distance Education
}

\author{
Xia Yaowen and Li Zhiping \\ Institute of Information \\ Yunnan Normal University \\ Kunming,Yunnan Province,China \\ xywen_km@sina.com
}

\author{
Lv Saidong and Tang Guohua \\ Institute of Security \\ Yunnan Normal University \\ Kunming,Yunnan Province,China \\ lsdong_km@sina.com
}

\begin{abstract}
- this paper analyzes the problems of heterogeneous databases in a distance education system. It provides a heterogeneous database integration model based on XML and design and develop a heterogeneous database integration platform based on this model. Thus, it provides a unified standard and effective tool for teaching resource description and management in distance education.
\end{abstract}

Keywords-distance education,heterogeneous database, XML

\section{INTRODUCTION}

The teaching resource types and the number of distance education are growing constantly with the rapid development of modern distance education. These resources are not standardized, dispersed and difficult to share and reuse because of these are not a unified description and management. So, in this case, there is need a method to integrate the heterogeneous data information with the rapid increase of teaching resources. This is a key issue that how to integrate and access these data. It is provided to the user a good interface to the user. The information is presented as a formatted data to the user, thus shielding the heterogeneous of these system platforms, operating environment and internal organizational structure. It enables the user operate any distance databases just like locally [1].

In order to solve the problem of how to describe and manage resources effectively, XML language has the characteristics of the excellent data storage format, scalable, highly structured and the convenience of network transmission. Thus, this paper will be provides an generic model based on XML, and applied to distance education system. It provides and effective tool for the description of the structure and management of the teaching content and teaching resource.

\section{ANALYSIS}

\section{A. Distance education platform heterogeneous}

The teaching resources heterogeneity of distance education platform can be roughly divided into the following three categories: operating environment heterogeneous, data storage type heterogeneous and logical relationship heterogeneous.

- The operating environment heterogeneous can be summarized in the difference that the operating system of the hardware environment, relevant data, control environment and communication environment.

- The data storage type heterogeneous refers to the differences between the storage systems of teaching resources in the entire education platform. The data resources of distance education platform are stored in SQL Server database system, but some functional modules may be relational database, such as Oracle,Access, Mysql ,etc.

- The logical relationship heterogeneous can be divided into related name of data elements, value represents the meaning,grammar,etc. The same value represents different semantics in a different operating environment and database system.

B. XML Middleware[2][3][4]

- The integration of cross-platform multi mode. XML has the characteristics of cross-platfrom,thus the heterogeneous database system based on XML can be facilitate transplantation between the various platforms. So that XML documents can be freely exchange data between the two different systems, it can be direct access to the heterogeneous databases.

- Solve the data structure conflicts between heterogeneous data sources. In this paper, it uses a two-layer model that global access and local access. The user's globally accessible decomposed into local access for each data source, Thereby shielding the data organization conflict of heterogeneous data sources.

- $\quad \mathrm{XML}$ and database interoperability. XML provides a universal, good data format of self-explanatory, the user access requests submitted to the database in the form of XML and database access results returned to the user in the from of XML. It is an ideal solution of the application development in the future.

C. The system demands of heterogeneous database in distance education

- a description function of the teaching resources

The XML language has good semantics and clear structure, so that the user can customize according to the specific circumstances of the mark. Teaching resource content tree hierarchy data representation and the XML hierarchical and structured document organization is just 
consistent, so we can use the XML language to describe the organizational structure of the teaching resources.

- a management function of the teaching resources

We can achieve the standardization of teaching resources through standardized XML language to define resources information format. In addition to it management standardized description of teaching resources, teaching resources and effective management to achieve teaching resources. Teaching resources, including various child resource views, add, modify, and delete operations. It's order is not static between teaching resources knowledge, it needs to organize the structure of resources dynamic based on XML. We can achieve a dynamic organization of teaching resources by adjust the XML document structure.

- a communication and transferring function of the teaching resources

$\mathrm{XML}$ is the ideal format for data exchange Internet on different systems on different platforms. The resources which is used standardized resource description format can exchange and transfer teaching resources. Thus it can be share and reuse the resource.

\section{THE DESIGN OF HETEROGENEOUS DATABASE}

\section{INTEGRATION SYSTEM IN DISTANCE EDUCATION PLATFORM}

The distance education management platform which is based on the ADL-SCORM standardization use XML language to descript the teaching resources, so that it can be achieved the standardization of the teaching resources and to validate the resource description document by XML Data Definition Language (XML Schema)[5].The teaching resources of distance education are stored in the library of different kinds of resources, such as audio resource library, video resource library, PPT resource library. These resource libraries are stored in the different resources on the server. Bind a variety of learning resources metadata by XML, and then parse these metadata, so that map it which is currently the most popular network standard metadata. It is stored in the local server [6]. In the resource description and management system, it has the different types of repository and XSL template library. XSL template library storage is applicable to different users, different needs of template XSL file. The client module is responsible for the loading the XSL template file of XSL template library, it provide to the users to personalize the user interface, respectively, and to achieve different functions. The purpose of the description and management in distance education resources is to achieve the standardization of teaching resources with XML language unified description of a wide range of diverse type of teaching resources, thus it manage resources effective. It makes sharing between heterogeneous systems and reuses the teaching resources by a method of this XML description resource metadata and uniform standards of its content structure. The architecture diagram of heterogeneous database integration platform is shown in Figure 1.

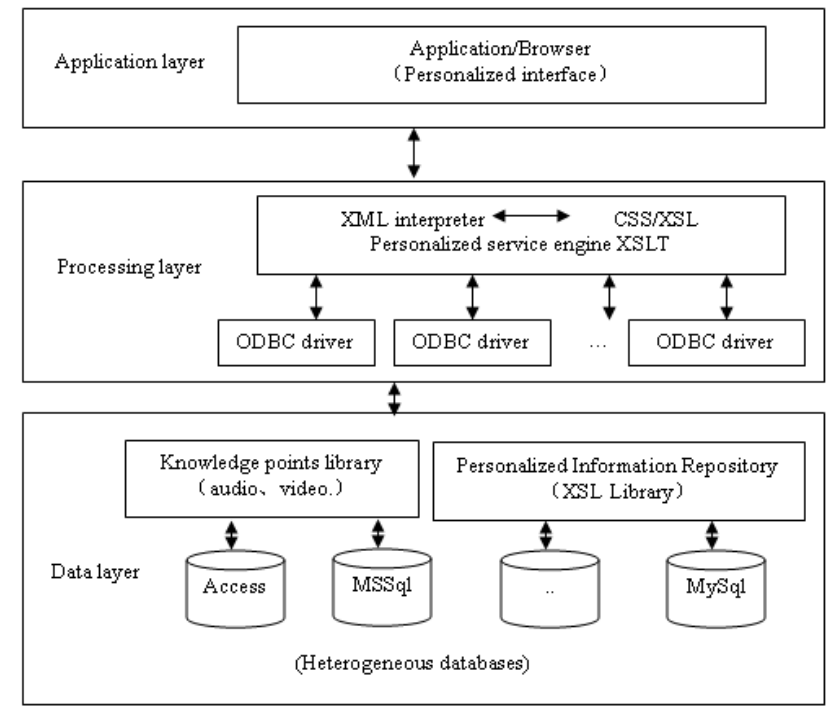

Figure 1. The architecture diagram of heterogeneous database integration platform

\section{THE IMPLEMENTATION PROCESS OF HETEROGENEOUS DATABASE}

In this paper, the idea is that through user input information thus query heterogeneous database, then traverse related database, take out data and these data in accordance with the structure of the query results organize into file which is expressed in XML structure. This process can be divided into two phases:

Early stage: A model of the establishment heterogeneous database integration;

Real-time stage: Accept the user's query and return query results.

The early stage consists of two steps:

- Establish global data dictionary which consists of a series of tables is the global resources of the entire heterogeneous database system.

- Create correspondence relationship between heterogeneous database and local database by the virtual database relations dictionary.

The late stage consists of four steps:'

- The user's standard query(XML format )translate into a sequence of heterogeneous database access;

- Convert the query access parameters and access heterogeneous databases;

- Get heterogeneous database SQL query results and convert into an XML document;

- Provided for each database query results which is that XML document integrated processing.

The implementation process of heterogeneous database is shown in Figure 2. 


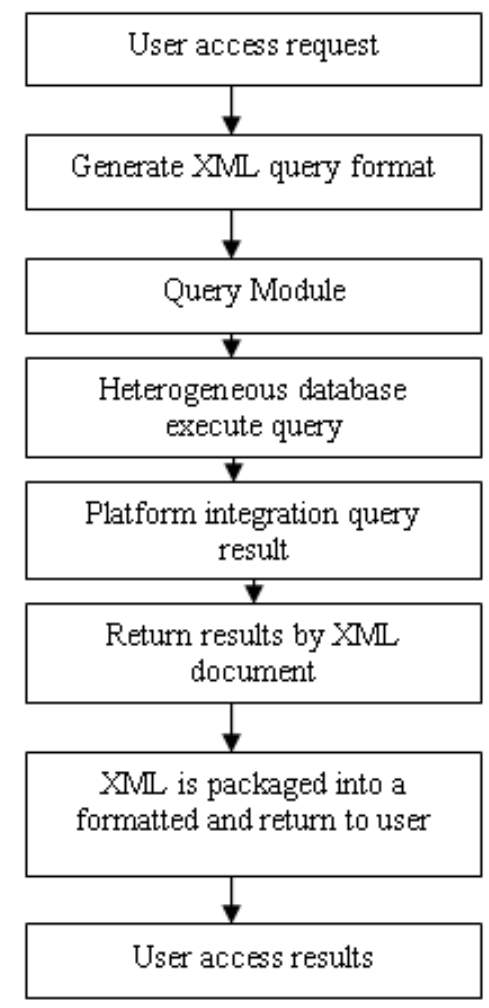

Figure 2. The implementation process of heterogeneous database

\section{THE IMPLEMENTATION OF SYSTEM}

Based on above ideas we develop heterogeneous database integration platform which is based on the Microsoft Visual Studio.NET Platform, using Visual C\# as development language and database is SQL Server 2005. This platform is shown in Figure 3, Figure 4 and Figure5.

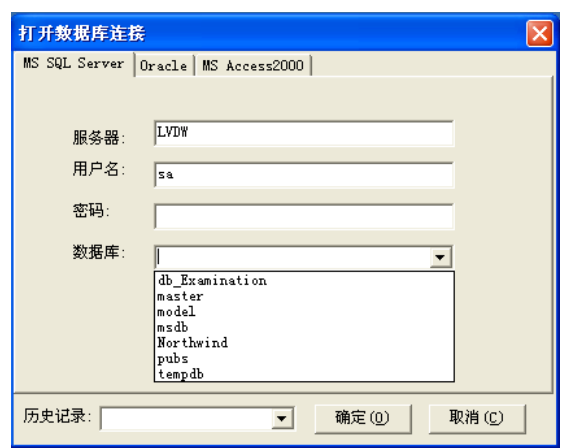

Figure 3. Heterogeneous database connection

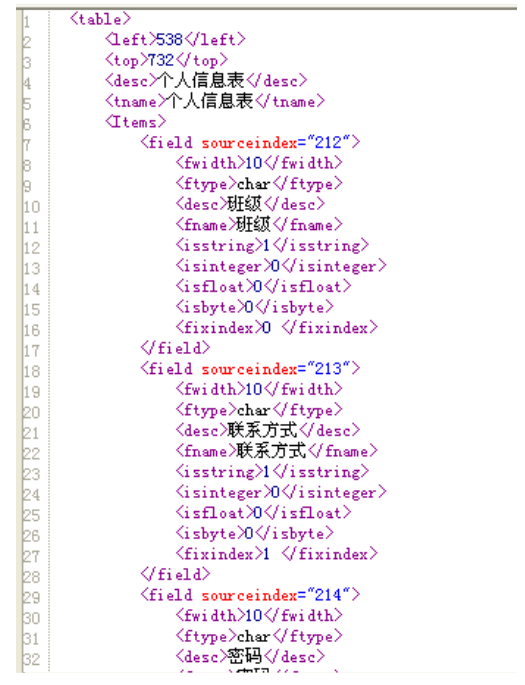

Figure 4. Convert into an XML document

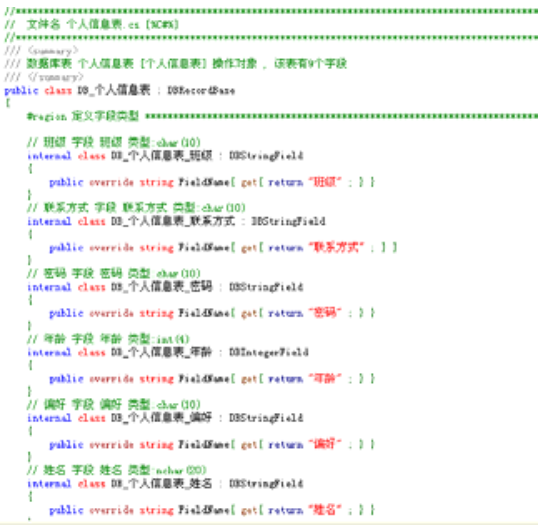

Figure 5. Convert into a simple Visual C\# code Its main teatures are the tollowing aspects:

A. The platform can extracted the model automatically from the existing database. It also can update model and apply to the database automatically by the tools and editor.

B. Database access results convert into XML document

The relational database data convert into an XML document, follow these steps:

- For each table in the database create its table name of the corresponding elements.

- For each column in the table create its attribute of the corresponding elements.

- For each table primary key/foreign key relationship create its child elements of the corresponding elements. Merge into an XML document between various elements and sub-elements based on the relationships between primary key and foreign key, and then optimize the XML document. 


\section{XML document Convert into the relationship database}

XML documents convert into the relationship database using the following mapping rules:

- For a single element of the XML which is directly mapped to the corresponding column in the table.

- $\quad$ For composite elements of the XML, the composite element attributes and child elements are mapped to the corresponding column in the table.

- For the composite sub-elements of the XML, the affiliation of its main elements linked by PK code.

\section{CONCLUSION}

In this paper analyze and research XML language and related technologies based on the requirement analysis of teaching resources and management in distance education. It provides a heterogeneous database integration model based on XML. Using Visual Studio.NET 2005 and SQL 2005 develop a heterogeneous platform based on this model of this paper provided. Last this platform is applied to the distance education system. In the future, it will be study on the combination of the technology and web, and to achieve cross-site intelligence sharing of information resources.

\section{ACKNOWLEDGMENT}

This work is supported by the National NSF of China (No.60903131), the Natural Science Fund (NSF) of Yunnan Province (No.2004F0017Q, 2005F0022Q, 2009ZC0052M).

\section{REFERENCES}

[1] IEEE Learning Technology Standards Committee [s]. http://ltsc.ieee.org

[2] Jia Xiaobao,Song Lizhi,Zhao Wei.Easy XML.First edition,Beijing: People Post Press,2001,1-8

[3] Geng Xiangyi,XML-based tutorial.First edition.Bejing:Tsinghua University Press,2006,2-9

[4] Chinese XML Forum, http://bbs.xml.org.cn/index.asp

[5] AAP KAMPS,MAARTEN MARX,MAARTEN DE RIJKE,BOERKUR SIGURBJOERNSSON.Articulating Information Needs in XML Query Languages.ACM Transactions on InformationSystems, 2006,24 (4) 407-436

[6] Kokou Yetongnon,Seksun Suwanmanee,Djamal Benslimane,PierreAntoine Champin.A web-centric semantic mediation approach for spatial information systems.Journal of Visual Languages\&Computing: 2006, 17 (1) $1-24$ 